[Deutsche Entomologische Zeitschrift XXIX. 1885. Heft II.]

\title{
Notizen über Rüsselkäfer
}

\author{
von \\ J. We is e.
}

1. Omias Hanaki Friv, zeichnet sich durch die sehr ungleichen Klauen vor den anderen Arten aus; die äufsere Klaue ist ziemlich lang, gebogen, die innere äufserst kurz und schwach. Bei O.concinnus und forticornis sind die am Grunde verwachsenen Klauen gleich lang. Das $\sigma^{\top}$ von concinnus hat erweiterte Tarsen und einen viel längeren letzten Bauchring als das $q$, der an der Spitze deutlich abgestutzt ist. Die auffälligen Geschlechtsunterschiede des Omias forticornis Boh., die ich weder von Seidlitz, noch sonst in der mir zugänglichen Literatur erwähnt finde (es sind als Unterschiede nur schmälere, gestrecktere Fld. und stark verdickte Schenkel beim Männchen genannt), bestehen darin, dafs beim $\sigma^{\uparrow}$ die Trochanteren der Vorderbeine in eine nach unten gebogene scharfe und lange Spitze ausgezogen sind, die dem $q$ fehlt, ferner besitzt der letzte Bauchring einen breiten, nach hinten erweiterten und an den Rändern mit längeren Haaren besetzten Längseindruck und ist in der Mitte des Hinterrandes in flachem Bogen ausgeschnitten. Bei den Weibchen ist der letzte Bauchring in der Mitte ziemlich eben, am Hinterrande tief, fast viereckig ausgeschnitten. Der Ausschnitt hat schwach ausgerandete Seiten und am Vorderrande ein nach hinten gerichtetes, fast dreieckiges, wahrscheinlich bewegliches Plättchen, welches nicht ganz so lang ist als der Ausschnitt selbst. Diese Bildung hat eine gewisse Aehnlichkeit mit der, welche beim $\sigma^{\top}$ der Anlacophora abdominalis auftritt. - Der Penis von forticornis ist lang und schmal, an der Basis schwach gerundet in die Höhe gebogen, davor geradlinig, allmählich in eine scharfe Spitze verschmälert.

2. Im Laufe der Zeit haben sich bei der Gattung Magdalis einige Unrichtigkeiten eingeschlichen, die einer Klarstellung bedürfen; ich gebe daher zunächst eine Uebersicht über die mir bekannten deutschen Arten und knüpfe die nöthigen Bemerkungen dahinter an. 


\section{Deutsche Magdalis-Arten.}

I. Klauen einfach.

1 Fühler dicht vor den Augen eingelenkt, die Fühlerfurche läuft am Vorderrande der Augen nach unten 13. nitidipennis Boh.

1' Die Fühlerfurche beginnt, von den Augen entfernt, in oder vor der Mitte des Rüssels

2 Fühlerschaft kurz, kaum doppelt so lang als das erste Geifselglied, in der oberen Hälfte etwas zusammengedrückt und stark erweitert . . . . . . 10. exarata Bris.

2' Fühlerschaft lang, vielmal länger als das erste Geifselglied 3 .

3 Vorderschenkel mit einem kleinen, undeutlichen Zahne, oder ungezähnt . . . . . . . . . . . . . . . 4 .

3' Die Vorderschenkel haben einen grofsen Zahn . . . . 6.

4 Oberseite rothbraun. Vorderschenkel undeutlich gezähnt.

4. $r u f a$ Germ.

4' Oberseite schwarz. Vorderschenkel ungezähnt . . . . 5.

5 Rüssel nur so lang als der Kopf, gerade. Seiten des Halssch. hinter der Mitte jederseits mit einem spitz gekörnten, hinten steil abfallenden Längshöcker 12. pruni L.

5' Rüssel länger als der Kopf, etwas gebogen. Seiten des Halssch. ohne Höcker . . . . . 11. barbicornis Latr.

6 Bauch äufserst dicht punktirt und sehr dicht, seidenschimmernd weifslich grau behaart. Augen gewölbt, fast halbkugelig . . . . . . . . . 3. phlegmatica Hbst.

6' Bauch sparsam behaart. Augen flach oder sehr wenig gewölbt

7 Die beiden ersten Bauchringe sind überall stark und tief punktirt, der Basaleindruck der Fld. reicht seitlich bis zum starken fünften Punktstreife

7' Die beiden ersten Bauchringe ganz oder wenigstens an den Seiten flach punktirt, der Quereindruck an der Basis der Fld. reicht seitlich über den fünften Punktstreifen hinaus

8 Augen sehr flach, bedeutend breiter als lang, mit den Schläfen in einem gleichmäfsigen Bogen bis an den Rüssel verlaufend; $Z$ wischenstreifen der grob punktirten Punktstreifen schmal, runzelig punktirt . . 1. memnonia Fald.

8' Augen schwach gewölbt, der mit den Schläfen gebildete Bogen krümmt sich vorn stark nach dem Rüssel heran; Zwischenstreifen der Fld. einreihig punktirt 2. linearis Gyllh. 
9 Punktstreifen 8 bis 10 der Fld. vor der Spitze auffällig stark vertieft, die Zwischenstreifen daselbst stark gewölbt, der 6. und 7. Punktstreif erlischt auf dem Abfalle zur Spitze in einer tiefen Grube, welche an der Vereinigung des 3. und 8. Streifens liegt und eine mälsig grofse, glänzendere Stelle davor höckerartig emporhebt. Stirn zwischen den Augen $\mathrm{schmal} \mathrm{.} \mathrm{.} \mathrm{.} \mathrm{.} \mathrm{.} \mathrm{.} \mathrm{6.} \mathrm{violacea} \mathbf{L}$.

9 ' Punktstreifen 8 bis 10 der Fld. vor der Spitze mäfsig vertieft, die Zwischenstreifen daselbst eben oder mäfsig gewölbt, der 6. und 7. Punktstreif sind bis zum Ende deutlich, kaum verfeinert, sie brechen plötzlich ab oder verbinden sich entweder beide mit einander oder mit dem 8 . Streifen, vor dessen Vereinigung mit dem 3. Streifen höchstens eine leichte Vertiefung auftritt . . . . . . . . . . 10.

10 Fld. mit schwachem Erzschimmer. Stirn zwischen den Augen mälsig breit . . . . . . . 5. nitida Gyllh.

$10^{\prime}$ Fld. ohne Spur von Erzschimmer. Stirn breit . . . . 11.

11 Punkte in den Zwischenstreifen der Fld. fein, von hinten eingestochen, vorn von einem glatten, glänzenden Körnchen begrenzt, hinten allmählich zur Fläche des Streifens ansteigend . . . . . . . 7. frontalis Gyllh.

11' Punkte in den $Z$ wischenstreifen von oben eingestochen, ihr Vorderrand kaum oder nur unbedeutend höher als der Hinterrand

12 Zwischenstreifen einreihig punktirt . . 8. duplicata Germ.

$12^{\prime}$ Die geraden $Z$ wischenstreifen einreihig, die ungeraden doppelreihig punktirt . . . . . 9. Weisei Schreiner.

II. Klauen gezähnt oder mit einer zahnartigen scharfen Erweiterung am Grunde.

1 Vorderschenkel mit grofsem dreieckigen Zahne . . . . 2.

1' - - undeutlichem Zahne oder ganz ungezähnt

2 Zwischenräume der Fld. fein lederartig gerunzelt, stark gewölbt, kaum so breit als die grob punktirten, tiefen Streifen. Halssch. mit 3 bis 5 stärkeren Körnchen jederseits hinter der Einschnürung . . . 14. carbonaria L.

2' Zwischenräume der Fld. deutlich breiter als die Streifen 3.

3 Der Fühlerschaft reicht ungefähr bis zur Mitte jedes Auges. Streifen der Fld. scharf, schmal, deutlich punktirt, Zwischenstreifen flach, breit, dicht und äufserst fein 
gerunzelt. Halssch. mit einem starken, spitzen Zahne hinter der Einschnürung jederseits . . 15. aterrima F.

3' Der Fühlerschaft reicht nur bis zum Vorderrande jedes Auges. Streifen der Fld. wenig scharf, sehr undeutlich punktirt, Zwischenstreifen ziemlich breit, leicht gewölbt, körnig-gerunzelt. Halssch. mit einer bogenförmigen, ziemlich gleich hohen Leiste jederseits hinter der Einschnürung.

16. asphultina Boh.

4 Vorderschenkel mit kleinem höckerförmigen Zähnchen. Augen lang-oval. Schildchen nach vorn abschüssig.

17. cerasi L.

4' Vorderschenkel ungezähnt oder, beim $\sigma^{\lambda}$, mit einem schwer sichtbaren spitzen Höckerchen. Augen kurz-oval. Schildchen horizontal.

5 Die Punktstreifen 6 und 7 der Fld. vereinigen sich hinten mit dem 8. Streifen; der zweite Zwischenraum von der Naht und vom Seitenrande ist an der Spitze stark gewölbt . . . . . . . . 18. flavicornis Gyllh.

5 ' Die Punktstreifen 6 und 7 der Fld, vereinigen sich hinten nie mit dem 8 . Streifen; der zweite $Z$ wischenraum von der Naht und vom Seitenrande ist an der Spitze nicht gewölbt oder in die Höhe getrieben . . . . 19. quercicola Ws.

Der allen Arten gemeinsame Geschlechtsunterschied besteht darin, dafs beim Weibchen der erste und zweite Bauchring gewölbt, beim Männchen in der Mitte der Länge nach flach gedrückt oder vertieft ist; zugleich ist bei den Männchen der meisten Arten die Behaarung einzelner oder aller Bauchringe auf einem breiten Streifen längs der Mitte aufgerichtet, was bei keinem Weibchen vorkommt. Letztere sperren nach dem Tode fast immer den Anus. Es empfiehlt sich daher, die Magdalis-Arten nur in der Berliner Manier, also mit dem Metasternum anzukleben, dagegen die bequemere Methode, bei der das Thier der Längsachse nach mit dem ganzen Bauche auf ein dreieckiges Papierstück gelegt wird, zu unterlassen.

Im Allgemeinen kommt die feinere Skulptur, namentlich der Fld, dem Männchen zu. Dafs der Mann einzelner Arten seltener zu sein scheint als das Weib, beruht wohl nur auf unserer mangelhaften Kenntnifs von der Flugzeit der Thiere.

1. Magd. memnonia Fald. Der Unterschied von linearis wird gewöhnlich in der verschiedenen Punktirung der Fld. an- 
gegeben; es kommen jedoch Stücke von linearis vor, die der memnonia so ähnlich werden, dafs man sie, ohne die Augenbildung zu berücksichtigen, davon kaum noch trennen kann. Das Männchen ist nur an der dichteren und halb a ufstehenden Behaarung über die Mitte aller Bauchringe zu erkennen. Der Penis ist sehr klein, schlank, ziemlich gleichbreit, an der Spitze abgerundet, oben mit einer Längsrinne versehen, die sich vorn erweitert und vertieft, so dafs die Spitze löffelförmig ausgehöhlt erscheint.

2. Magd. linearis Gyllb. $\sigma^{7}$. Die Mitte aller Bauchringe, namentlich der drei letzten, lang aufstehend behaart.

3. M. phlegmatica Hbst. $\sigma^{\nearrow}$. Alle Bauchringe, besonders die drei letzten, in der Mitte lang halb-aufstehend behaart. Penis schlank, an der Oeffnung allmählich etwas verengt, vorn abgerundet, die gerade abgestutzte Klappe der Oeffnung dreirinnig.

4. M. rufa Germ. $\sigma^{\top}$. Die letzten Bauchringe in der Mitte länger aufstehend behaart als an den Seiten.

5. M. nitida Gyllb. ${ }^{\Uparrow}$. Die drei letzten Bauchringe dichter behaart als beim $q$, die Behaarung der beiden ersten Ringe hoch, der drei letzten wenig aufgerichtet; der Rüssel merklich dicker als beim 오.

6. M. violacea L. Die Art ist hauptsächlich in den Alpen zu Hause, überhaupt in Oesterreich eine der häufigeren, aber auch um den Mt. Rosa und Mt. Viso, in Ungarn, Schlesien, der Mark Brandenburg, Mecklenburg (Habelmann), Hessen, Schweden etc. verbreitet; nur mit frontalis zu verwechseln, aber durch den kürzeren Rüssel, die schmale Stirn, welche zwischen den Augen etwa nur die Hälfte von der Breite der Rüsselbasis besitzt und über den Augen sparsamer punktirt und glänzender ist, das längere Halssch., die tieferen, schärfer begrenzten Punkte in den Streifen der Fld, von denen der neunte vorn, wo er unter der Schulterbeule schräg aufwärts biegt, auffällig breit und tief ist, sowie durch das allmähliche Erlöschen des 6. und 7. Streifens in der tiefen Grube auf dem Abfalle zur Spitze sicher zu unterscheiden. Beide Geschlechter sind viel mehr als bei den vorhergehenden und den drei folgenden Arten verschieden. Beim Männchen ist der Rüssel wenig gebogen, äufserst dicht gewirkt, matt, verloschen punktirt, der erste und zweite Bauchring in der Mitte aufstehend behaart, $=$ Heydeni Desbroch., violacea Seidl. F. balt. 430; beim Weibchen (cyanea Seidl. 1. c.) ist der Rüssel etwas länger, ziemlich gleichbreit und gleichdick, kräftig gebogen, glänzend, höchstens an der Basis gewirkt, fein, doch scharf punktirt. 
Die Art ist 3.3 bis 6.2 mill. lang (vom Hinterrande der Fld. bis zum Vorderrande der Augen gemessen) und variirt, wie die übrigen Arten in der Körperform, Farbe und Skulptur ganz erheblich. Schwarze Exemplare, die allerdings nur vereinzelt vorkommen, können nach den Bestimmungstabellen von Desbrochers und Seidlitz nicht untergebracht werden.

Ob M. coeruleipennis Desbr., weil sie einen stark gerundetvorgezogenen Grundrand der Fld. besitzen soll, wirklich specifisch von dieser Art verschieden sei, wofür sich Herr Czwalina in der Deutschen Ent. Zeitschr. 1884, p. 415, ausspricht, ist wohl so lange zu bezweifeln, bis andere Unterschiede erbracht worden sind, die der Täuschung weniger unterliegen.

7. M. frontalis Gyllh. Das Halssch. ist höchstens so lang als breit, in der Regel quer, jedoch niemals merklich länger als breit wie das der vorigen, zugleich ist die Stirn matter, dichter und deutlicher punktirt, der Grundrand der Fld. gewöhnlich mehr gerundet und stärker über die Basis des Halssch. vorgezogen; die Grölse variirt von 4 bis 7 mill., die Farbe von blau durch violettschwarz bis schwarz. Beide Geschlechter sind wenig verschieden. Der Rüssel ist eben so stark gebogen wie beim von violacea, beim $\sigma^{\top}$, das man nur an dem in der Mitte aufstehend behaarten ersten und zweiten Bauchringe mit Sicherheit erkennen kann, ist er vor der Fühlerwurzel unmerklich flach gedrückt und erweitert.

8. M. duplicata Germ. Das Männchen (=M. striatula Desbr.) zeichnet sich durch die aufstehende Behaarung der beiden ersten Hinterleibssegmente und den von der Basis zur Spitze allmählich, jedoch nicht besonders stark erweiterten Rüssel aus.

Die Art, welche 2.5-4.8 mill. lang wird, ist an der Punktirung der Fld., auf denen die $Z$ wischenstreifen eine regelmäfsige oder unregelmälsig verdoppelte Reihe von Punkten.besitzen, die von oben eingestochen sind, sicherer noch an der Bildung des Halssch. von der vorigen zu unterscheiden. Während sich hier das Halssch. hinter der Mitte bis an den Hinterrand verschmälert, an dem die Hinterecken in Gestalt einer schmalen, aber ziemlich hohen Querleiste plötzlich nach aufsen vortreten, endet die Verschmälerung bei frontalis ein Stück vor dem Hinterrande und die Seiten erweitern sich dahinter allmählich wieder zu den dreieckigen Hinterecken, welche, auch bei der Betrachtung von unten, in keine deutlich aufgebogene Querleiste auslaufen. - Schwarze Stücke sind nicht selten. 
9. Von M. Weisei Schreiner ist mir das $\sigma^{\top}$ noch unbekannt. Einer Vereinigung mit virescens Germar steht hauptsächlich die Angabe: "rostrum parum curvatum" im Wege.

10. M. exarata Bris. Das Männchen, von mir früher als M. Kratzi beschrieben, hat eine ähnliche Fühlerbildung wie das von barbicornis und cerasi, indem die drei letzten Fühlerglieder in eine langgestreckte, dicht bürstenartig behaarte Keule verlängert sind, welche länger ist, als die übrigen Geifselglieder zusammen. Von den Keulengliedern ist das erste kurz, das zweite nach oben verbreitert und hier etwas breiter als 1 , das dritte ist doppelt so lang als die beiden vorhergehenden zusammen, in der Mitte etwas eingeschnürt.

Ich sammelte das Thier noch in der Mark Brandenburg auf Eichen bei der Buschmühle bei Frankfurt a. O., besitze es aufserdem aus Ungarn und Griechenland (Reitter).

11. M. barbicornis Latr. Beim $\sigma^{\top},=$ claviger Küst., ist das 8. Fühlerglied zwar von der walzenförmigen Keule deutlich getrennt, aber eben so breit als das erste Glied derselben und auch eben so dicht, abstehend behaart; das zweite Keulenglied ist etwa doppelt so lang als das erste, das dritte doppelt so lang als das zweite. - Der Penis ist vorn allmählich stark verbreitert, an der Spitze in breitem Bogen abgerundet, die Oeffnung theilweise von der gerade abgestutzten Klappe bedeckt.

Bei welcher Art M. mixta und turcica Desbr. unterzubringen sind, habe ich aus der Beschreibung nicht feststellen können.

12. M. pruni L. Zuweilen verschwindet der Höcker an den Seiten des Halssch. vollständig. Beide Geschlechter sind wenig verschieden. Der Rüssel ist beim $q$ wenigstens in der vorderen Hälfte glänzend, doppelt so lang als dick, beim $\sigma^{\uparrow}$ kurz, matt, kaum länger als dick. - Penis vorn allmählich in eine lange und schmale, am Ende schwach abgestutzte Spitze verschmälert.

13. M. nitidipennis Boh. Das $\sigma^{\top}$ ist mit Sicherheit nur an dem flachen Bauche zu erkennen.

14. M. carbonaria L. In seltenen Fällen sind die Körnchen hinter der Einschnürung des Halssch. nicht zu bemerken (ein Ex. in der Kalkhorst in Mecklenburg-Strelitz von Hrn. Habelmann gefangen). Beide Geschlechter sehr verschieden. Das $\sigma^{\top}$ hat eine aufstehende Behaarung auf den ersten beiden Bauchringen, einen sehr dicken, fast kantigen Rüssel mit einer auffällig breiten und tiefen Fühlerfurche und dicke Fühler. An diesen ist besonders der Schaft verdickt, beinahe dreimal so dick als beim Weibchen. 
15. M. aterrima F. Die Geschlechtsunterschiede sind dieselben, wie bei der vorigen Art, nur eine Spur schwächer ausgeprägt.

16. M. asphaltina Boh. Die Artrechte wurden erst wieder durch Hrn. Habelmann (Berl. Ent. Zeitschr. 1882, p. 391-394) festgestellt, nachdem das in den meisten Sammlungen fehlende Thier lange Zeit mit den vorigen Arten verbunden worden war. Das $\sigma^{\top}$ hat einen kurzen, dicken, in der vorderen Hälfte oben flachgedrückten Rüssel und viel dickere Fühler als das ; das letzte Segment ist, wenigstens an den von Hrn. Brenning in Wittenberg gezogenen Stücken, auffällig lang weifslich behaart und am Hinterrande jederseits in eine stumpfe Ecke aufgebogen. Im Freien gefangene Exemplare besitzen wahrscheinlich nur eine schwache, kurze Behaarung auf dem letzten Bauchringe.

17. M. cerasi L. Beim $\sigma^{\pi}=$ rhina Gyllh., ist der Bauch in der Mitte länger aufstehend behaart, der kurze, glanzlose Rüssel vorn breit und dick, die Fühler sind merklich dicker als beim $q$, die dreigliederige Keule ist sehr lang elliptisch, das erste Glied derselben ist das kürzeste, das letzte das längste.

18. M. flavicornis Gyllh. $\sigma^{\nearrow}$. Rüssel kürzer und um die Hälfte dicker als beim $q$, die Fühler viel kräftiger, der Schaft wenig und fast gleichmälsig gebogen, beim + dagegen dicht an den Augen stark und ziemlich schnell nach aufsen gekrümmt.

19. M. quercicola Ws. Die hauptsächlichsten Unterschiede von der vorigen Art bestehen in Folgendem: Das Halssch. ist bedeutend tiefer punktirt, an den Seiten gleichmälsiger gerundet, vorn und hinten weniger eingeschnürt-verengt. Die Streifen der Fld. sind stärker und schärfer punktirt, aber nicht so tief ein-

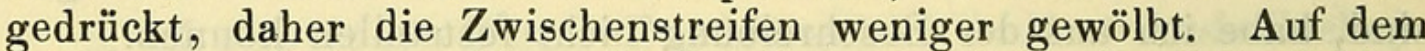
Abfalle zur Spitze endigen die Streifen 4 bis 7 in ziemlich gleicher Länge, entweder unverbunden oder zu zweien, dreien oder vieren verbunden, dadurch entsteht eine ziemlich ebene, dreieckige Stelle hinter ihnen und vor der Vereinigung des 3. und 8. Streifens, die nur gerunzelt ist. Diese Stelle fehlt bei flavicornis, da sie von dem tiefen 6. und 7. Streifen durchschnitten wird, die sich, weit hinter dem 4. und 5. Streifen, mit dem achten vereinigen. An den Fühlern ist der Schaft, nebst den zwei ersten Geifselgliedern röthlich gelb, der übrige Theil bei ausgefärbten Stücken immer dunkel, die Geifselglieder haben eine schmale Basis, so dafs sie viel deutlicher von einander abgesetzt sind wie bei flavicornis.

Beide Arten finden sich bei Berlin im Juni in ziemlicher Menge auf Eichen bei Pankow.

Fraglich blieb mir zuletzt noch M. punctulata Muls., weil alle unter dieser Bezeichnung erhaltenen Stücke sich als schwarze frontalis und violacea herausstellten. 


\section{$2 \mathrm{BHL}$ Biodiversity Heritage Library}

Weise, Julius. 1885. "Notizen über Rüsselkäfer." Deutsche entomologische Zeitschrift 1885(2), 409-416. https://doi.org/10.1002/mmnd.48018850235.

View This Item Online: https://www.biodiversitylibrary.org/item/103411

DOI: https://doi.org/10.1002/mmnd.48018850235

Permalink: https://www.biodiversitylibrary.org/partpdf/235357

\section{Holding Institution}

Harvard University, Museum of Comparative Zoology, Ernst Mayr Library

\section{Sponsored by}

Harvard University, Museum of Comparative Zoology, Ernst Mayr Library

\section{Copyright \& Reuse}

Copyright Status: Public domain. The BHL considers that this work is no longer under copyright protection.

This document was created from content at the Biodiversity Heritage Library, the world's largest open access digital library for biodiversity literature and archives. Visit BHL at https://www.biodiversitylibrary.org. 